\title{
A situação do câncer gástrico em Salvador, no Brasil e no mundo
}

\author{
The situation of gastric cancer in Salvador, in Brazil and in the world \\ La situación del cáncer gástrico en Salvador, Brasil y el mundo
}

Gabriela Romão de Almeida Carvalho Santos ${ }^{1 *}$, Jéssica dos Santos Gomes ${ }^{1}$, Karla Alessandra Mesquita Pereira ${ }^{1}$, Brenda dos Anjos Tosta da Silva ${ }^{1}$, Ruama de Souza Nogueira ${ }^{1}$, Adriele de Santana dos Santos ${ }^{1}$, Victória Almeida Santos Nascimento ${ }^{1}$, Crislaine Silva de Oliveira ${ }^{1}$, Tyciana Paolilo Borges ${ }^{1}$, Letícia Cabral Domingos da Rosa1.

\section{RESUMO}

Objetivo: Verificar através de uma revisão narrativa a respeito da taxa de incidência do câncer gástrico em Salvador, no Brasil e no mundo, identificar quais são as terapêuticas atuais e disponíveis e quais são os prognósticos recentes. Revisão bibliográfica: De acordo com a análise dos dados encontrados, a videolaparoscopia associada à quimioterapia e/ou a radioterapia são as mais utilizadas atualmente no tratamento desta patologia, afetando positivamente o prognóstico dos indivíduos, em torno do 5 ano de vida, e melhorando a sobrevida destes pacientes. A incidência do câncer gástrico, apesar de ocupar o 5 lugar no ranking de neoplasia mais comum no mundo, vem reduzindo sua taxa de morbimortalidade. Considerações finais: Compreende-se a necessidade de melhora no estadiamento do câncer gástrico, a fim de melhorar as respostas terapêuticas, aumentando o prognóstico da sobrevida destes indivíduos para além dos 5 anos, garantindo uma melhor assistência da equipe de saúde e reduzindo o número de óbitos causados por esta doença.

Palavras-chaves: Câncer, Estômago, Tratamento, Prognóstico.

\section{ABSTRACT}

Objective: To verify through a narrative review about the incidence rate of gastric cancer in Salvador, Brazil, and the world, to identify what are the current and available therapies and what are the recent prognosis. Bibliographic review: According to the analysis of the data found, videolaparoscopy associated with chemotherapy and/or radiotherapy are the most used methods today in the treatment of this pathology, positively affecting the prognosis of individuals, around 5 years of life, and improving the survival of these patients. The incidence of gastric cancer, despite occupying the 5th place in the ranking of the most common neoplasia in the world, has been reducing its rate of morbidity and mortality. Final considerations: The need to improve the staging of gastric cancer is understood in order to improve therapeutic responses, increasing the prognosis of survival of these individuals beyond 5 years, ensuring better assistance from the health team and reducing the number of deaths caused by this disease.

Key words: Cancer, Stomach, Treatment, Prognosis.

\section{RESUMEN}

Objetivo: Constatar a través de una revisión narrativa a respecto de la tasa de incidencia del cáncer gástrico en Salvador, en el Brasil y en el mundo, identificar cuáles son las terapias actuales y disponibles y los pronósticos recientes. Revisión bibliográfica: De acuerdo com la análisis de los dados encuentrados, la vídeo laparoscopia asociada con la quimioterapia y/o la radioterapia es la más utilizadas actualmente en tratamiento de esta patología, afectando positivamente el pronóstico de los individuos, alrededor de los 5 años de vida, y mejorando la supervivencia de estos pacientes. La incidencia del câncer gástrico, a pesar de ocupar el 5 l lugar en el ranking de neoplasia más comunes del mundo, viene reduciendos tasa de morbimortalidad. Consideraciones Finales: Se entiende necesidad de mejora en estadificación del câncer gástrico, a fin de mejorar las respuestas terapêuticas, aumentar el pronóstico de la supervivencia de estas personas hasta allá de los 5 años, a fin de garantizar una mejor asistencia del equipo de salud y reducir el número de muertes causadas por esta enfermedad.

Palabras clave: Cáncer, Estômago, Tratamiento, Pronóstico.

1Universidade Salvador (UNIFACS), Salvador - BA. *E-mail: gabrielaromaoalmeida98@gmail.com 


\section{INTRODUÇÃO}

O câncer gástrico, como o próprio nome referência, é o câncer que acontece em um dos principais órgãos do sistema digestivo, o estômago. É neste órgão onde ocorre o início da digestão do bolo alimentar e a produção do suco gástrico através das glândulas gástricas. Dados apontam que a neoplasia que ocupou o quinto lugar no mundo em 2012 foi à neoplasia gástrica, com quase um milhão de casos, totalizando a taxa de $6,8 \%$ (SANTOS MO, 2018).

A sintomatologia da neoplasia no estômago geralmente não é específica, pois pode ser confundida com outras patologias; porém, existem alguns sinais que fazem suspeitar desta patologia como perda de apetite, sensação de estômago cheio, perda de peso, vômitos, fadiga, sangramento gástrico através das fezes ou do vômito e dor à palpação no epigástrio durante exame físico (INCA, 2018).

O diagnóstico da neoplasia gástrica tem progredido substancialmente nos últimos anos, devido à maior qualificação médica, propiciando um tratamento de melhor qualidade, com diminuição do índice de mortalidade e aumento da qualidade de vida e sobrevida destes pacientes (FERLAY J, et al., 2015). Pode ocorrer de três formas: leiomiossarcoma, adenocarcinoma e linfoma, cada um com sua particularidade fisiopatológica que irá definir qual terapêutica deverá ser adotada para melhor atender cada caso em particular (INCA, 2018).

Variados são os fatores de risco para a neoplasia gástrica, todavia, a infecção por Helicobacter Pylori $(H$. Pylori), é umas das causas que mais se relaciona ao desenvolvimento dessa patologia (CHANG WK, et al., 2001; DIACONU S, et al., 2017; STEWART BW e WILD CP, 2014). Fatores como hábitos alimentares, o exacerbado consumo de bebidas alcoólicas, tabagismo e obesidade também estão associados ao aparecimento do câncer gástrico. Em contrapartida, refere-se que certos tipos de alimentos como, por exemplo, frutas, verduras, frutos do mar e cereais auxiliam no combate a esta doença; ou seja, estes alimentos podem assessorar o organismo como mecanismo de proteção (STEWART BW e WILD CP, 2014; WANG Q, et al., 2014).

No ano de 2015, somente no Brasil, o câncer de estômago foi responsável por 14.264 casos, sendo 9.132 homens e 5.132 mulheres. Para 2016-2017, a estimativa foi de 20.520 novos casos de câncer de estômago, sendo 12.920 homens e 7.600 mulheres, de acordo com o Instituto Nacional de Câncer. A estimativa para os anos de 2018 - 2019 foram de 21.290 casos, sendo 13.540 homens e 7.750 mulheres no Brasil. Em 2018, a estimativa foi de 1.033 .701 novos casos (SANTOS MO, 2018).

O presente estudo justifica-se pelo fato do impacto da grande incidência do câncer gástrico na sociedade atual. Em função disso, torna-se relevante analisar o panorama atual desta patologia, que continua ceifando tantas vidas e/ou implicando em muitos gastos para a saúde pública.

Portanto, objetivou-se verificar através de uma revisão narrativa a respeito da taxa de incidência do câncer gástrico em Salvador, no Brasil e no mundo, além de identificar as terapêuticas atuais e disponíveis e os prognósticos recentes.

\section{REVISÃO BIBLIOGRÁFICA}

\section{Etiologia do câncer gástrico}

No Brasil, a prevalência do diagnóstico de câncer de estômago ocorre no estágio avançado, tornando-se relevante a descoberta precoce das principais causas desta patologia. Carvalho JB, et al. (2010) afirma que o câncer gástrico é uma patologia de etiologia multifatorial, com o desenvolvimento de tumores a partir de lesões na mucosa gástrica, desencadeadas pela ação e/ou interação de fatores de risco, ou seja, pela exposição a fatores endógenos (genéticos) e exógenos (ambientais), por um longo período de tempo.

As alterações no material genético são representadas pelos fatores endógenos, no qual se destacam no desenvolvimento e progressão do câncer gástrico (RIBEIRO HF, et al., 2010). Em um processo cancerígeno, a célula sofre mutações genéticas, adquirindo instruções modificadas e desenvolvendo funções erradas. $O$ câncer pode se originar de duas formas, através dos oncogenes ou dos genes supressores do tumor (INCA, 2018). 
A H. pylori apresenta $75 \%$ das causas em todo o mundo envolvendo a gênese do câncer gástrico, sendo considerada cancerígena tipo I desde 1994. Há também evidências de que a infecção por H. pylori é uma condição necessária, mas não suficiente para explicar a carcinogênese gástrica (GONZÁLEZ CA e AGUDO A, 2012). Apesar da alta prevalência de infecção por $\mathrm{H}$. pylori nos casos de câncer gástrico, dos 100 infectados, apenas 1 irá desenvolvê-la (YOON H e KIM N, 2015)

Como fatores de risco, predomina-se o tabagismo e a ingestão de álcool. A formação endógena de nitrosamina e dieta podem estar envolvidos na causalidade do câncer gástrico e $11 \%$ a $18 \%$ dos casos desse tipo de câncer estão associados ao tabagismo. Além disso, existem evidências de que a ingestão de frutas e vegetais está associada a uma diminuição do risco para essa doença (GONZÁLEZ CA e AGUDO A, 2012). O histórico familiar também pode determinar o aparecimento do câncer gástrico (YAGHOOBI M, et al., 2017). Outros fatores de risco são citados, como idade acima de 70 anos, prevalência no sexo masculino, relação com patologias gástricas e uso exacerbado de tabaco e outras substâncias tóxicas (BAÚ FC e HUTH A, 2011).

\section{Diagnóstico do câncer gástrico}

O diagnóstico desta patologia se dá em função da sintomatologia apresentada pelo paciente, sendo confirmada através de exames de imagem. Na maioria das vezes, é detectada através dos pólipos gástricos, por meio de endoscopia digestiva, que permite a visualização de lesões e anomalias no estômago. Por meio da biópsia e/ou exame radiológico contrastado do estômago é possível realizar a avaliação citológica, possibilitando a obtenção de imagens que permitem verificar a presença de possíveis anomalias e tumores (INCA, 2018).

A complexidade do diagnóstico das neoplasias é devido às suas localizações variadas. Quando o diagnóstico é tardio, ocorrerá uma menor probabilidade de sucesso no tratamento e menor taxa de sobrevivência ao câncer. Cerca de menos de $30 \%$ dos pacientes diagnosticados no estado avançado tem sobrevida maior a 5 anos (STEWART BW e WILD CP, 2014),

Na maioria das vezes, o diagnóstico é estabelecido por médicos que não possuem especialização na área de oncologia. Este fato ocorre, pois em muitas ocasiões os pacientes não sabem a natureza da sua doença e, consequentemente, não procuram profissionais especializados na área (INCA, 2018).

\section{Incidência do câncer gástrico}

Observou-se que o câncer gástrico ocupa o 5 ํ lugar no ranking mundial de casos no mundo, chegando $6,8 \%$ de todos os casos (FORD AC, et al., 2015). Em relação ao número de mortes a neoplasia de estômago encontra-se em $3^{\circ}$ lugar em todo o mundo $(8,2 \%)$, perdendo apenas para o câncer de pulmão e câncer de colorretal. Em todo o mundo, essas taxas estão caindo devido à crescente aquisição de uma dieta rica em nutrientes e vitaminas presentes em frutas, legumes, vegetais, e uma dieta pobre em sal e alimentos conservados no mesmo (SHELLEY M, 2016).

No estudo feito por Ferlay J, et al. (2015), foram notificados 14,1 milhões de novos casos de câncer no mundo, sendo que houve 8,2 milhões de mortes causadas pelo mesmo. Neste mesmo ano foi verificado que o câncer que mais matava foi o de pulmão, tanto em número de mortes, 19,4\% do total, como em número de casos, $12,9 \%$ do total.

Conforme a base de dados Global Cancer Observatory (GLOBOCAN), o câncer gástrico está ocupando o quinto lugar de neoplasia mais comum no mundo. As cinco principais neoplasias incidentes no mundo são pulmão, mama, colorretal, próstata e estômago, nessa ordem respectivamente. A estimativa é de 1.033.701 novos casos de câncer gástrico no mundo, abrangendo todos os continentes: Ásia, Oceania, América Latina e Caribe, Europa, África e América do Norte (WHO, 2018).

O continente com maior incidência deste tipo de câncer é o Asiático, sendo responsável por $74,5 \%$, ou seja, 769.728 novos casos. O continente Europeu é o segundo com maior incidência, responsável por 12,4\%, 133.133 novos casos, seguida da América Latina, com 6,5\%, 67.058 novos casos, a África com 31.148 (3\%) novos casos, a América do Norte com 29.275 (2,8\%) novos casos e a Oceania com 3.359 novos casos $(0,32 \%)$, sendo o continente com maior incidência da doença (WHO, 2018). 
Segundo o INCA (2018), chegaram ao número de 9.132 mortes em homens e 5.132 nas mulheres causadas pelo câncer de estômago no Brasil (SHELLEY M, 2016). A estimativa da ocorrência do câncer gástrico no ano de 2018, no Brasil, serviu essencialmente para nortear quais regiões iriam necessitar de maior atenção, devido a uma elevada estimativa de aparição de casos de câncer gástrico.

Entre os estados brasileiros. As regiões onde a incidência do câncer gástrico mais tem impacto são Norte e Nordeste, sendo o segundo câncer mais frequente em homens. Nas regiões Sul e Centro-Oeste, este tipo de câncer ocupa o quarto lugar, em homens. Na região Sudeste, é o quinto mais frequente em homens. Em mulheres, nas regiões Sul, Centro-Oeste e Norte, esta neoplasia ocupa o quinto lugar. Na região Nordeste, é o sexto mais frequente e no Sudeste, fica em sétima posição (SANTOS MO, 2018).

De acordo a estimativa do INCA (2018), a segunda região com maior predominância do câncer gástrico é o Nordeste. Os estados mais acometidos por esta patologia, no Nordeste, são: Ceará $18,11 \%$ em homens e $11,13 \%$ em mulheres; na sua capital Fortaleza 12,8\% em homens e 10,01\% em mulheres. No Rio Grande do Norte para homens $14,12 \%$ e mulheres $8,93 \%$; na sua capital Natal $13,19 \%$ para homens e $8,77 \%$ nas mulheres. Na Paraíba 13,69\% em homens e 9,41\% em mulheres; em João Pessoa $13,29 \%$ em homens e $7,63 \%$ em mulheres. No estado de Pernambuco $11,88 \%$ em homens e 7,68\% em mulheres e em sua capital Recife as taxas para homens é de $12,13 \%$ e em mulheres $8,59 \%$. Na Bahia as taxas são de $9,15 \%$ para homens e 6,19\% para mulheres; sendo que em Salvador essas taxas são de $11,52 \%$ em homens e $8,43 \%$ em mulheres (SANTOS MO, 2018).

Tendo em vista dados apontados neste estudo, percebe-se um alto índice de casos de câncer no estômago na região Nordeste, sendo o segundo mais frequente em homens. No estado da Bahia essa neoplasia é a quarta mais frequente entre homens (SANTOS MO, 2018).

O primeiro continente com maior estimativa de novos casos em 2018, é o continente asiático, responsável por 769.728 novos casos de câncer gástrico, que são distribuídos principalmente entre China, Japão, Índia, República da Coréia e Vietnã, respectivamente nesta ordem. Estes cinco países, atualmente, são responsáveis por $88,9 \%$ dos casos de câncer de estômago na Ásia, os $11,1 \%$ restantes são repartidos nas demais regiões (WHO, 2018).

O segundo continente com maior estimativa de novos casos em 2018, é o europeu, responsável por 133.133 novos casos de câncer gástrico, que são distribuídos principalmente entre Federação Russa, Alemanha, Itália, Ucrânia e França, respectivamente nesta ordem. Estes cinco países, atualmente, são responsáveis por $59,4 \%$ dos casos de câncer de estômago na Europa, os 40,4\% restantes são repartidos nas demais regiões (WHO, 2018).

O terceiro continente com maior estimativa de novos casos em 2018, é o Latino Americano e Caribe, responsável por 67.058 novos casos de câncer gástrico, que são distribuídos principalmente entre Brasil, México, Colômbia, Peru e Chile, respectivamente nesta ordem. Estes cinco países, atualmente, são responsáveis por $69,8 \%$ dos casos de câncer de estômago na América Latina e Caribe, os 30,2\% restantes são repartidos nas demais regiões (WHO, 2018).

O quarto continente com maior estimativa de novos casos em 2018, é o Africano, responsável por 31.148 novos casos de câncer gástrico, que são distribuídos principalmente entre República Democrática do Congo, Nigéria, Egito, Argélia e Quênia, respectivamente nesta ordem. Estes cinco países, atualmente, são responsáveis por $38,4 \%$ dos casos de câncer de estômago na África, os $61,6 \%$ restantes são repartidos nas demais regiões (WHO, 2018).

O quinto continente com maior estimativa de novos casos em 2018, é o Norte Americano, responsável por 29.275 novos casos de câncer gástrico, que são distribuídos principalmente entre Estados Unidos e Canadá, respectivamente nesta ordem. Estes dois países, atualmente, são responsáveis por $99,98 \%$ dos casos de câncer de estômago na América do Norte, os 0,02\% restantes são repartidos nas demais regiões (WHO, 2018).

O sexto continente com maior estimativa de novos casos em 2018, é o continente da Oceania, responsável por 3.359 novos casos de câncer gástrico, que são distribuídos principalmente entre Austrália; 
Papua Nova Guiné; Nova Zelândia; França, Nova Caledônia e Fiji, respectivamente nesta ordem. Estes cinco países, atualmente, são responsáveis por $97,4 \%$ dos casos de câncer de estômago na Oceania, os 2,6\% restantes são repartidos nas demais regiões (WHO, 2018).

\section{Tratamento e prognóstico do câncer gástrico}

O câncer gástrico, apesar de ter reduzido sua incidência de forma considerável, principalmente em países desenvolvidos, ainda possui um diagnóstico tardio, limitando o seu tratamento (SONG H, et al., 2016). O tratamento para essa patologia pode ocorrer por meio de: cirurgia, quimioterapia e radioterapia. A terapia a ser escolhida é definida quando se realiza uma análise sobre o tipo histológico, o tamanho, a localização e a extensão deste câncer.

Após o término dessa análise, será definida qual a melhor abordagem terapêutica. A cirurgia é a retirada de forma parcial ou total da parte afetada do estômago pela doença. A quimioterapia é a aplicação de compostos químicos no organismo com a finalidade de destruir as células malignas e a radioterapia é a utilização de radiações que destroem ou simplesmente impedem que as células malignas se proliferem (OMS, 2018).

Segundo a Comissão Nacional de Incorporação de Tecnologias no SUS (2017), o prognóstico e tratamento do câncer gástrico será definido pela localização e estadiamento do tumor, número de linfonodos ressecados acometidos e a presença de metástases. Responsável pela tomada de decisões sobre o tratamento a ser aplicado, a equipe multidisciplinar deve identificar e avaliar o histórico do paciente, evolução do tumor com base na sua agressividade, possibilidade de doença residual, riscos cirúrgicos e a resposta do paciente a tratamentos não isentos de efeitos colaterais (TONETO MG e VIOLA L, 2018).

O estudo feito por Toneto MG e Viola L (2018), mostrou que os tratamentos comumente utilizados são: laparotomia, laparoscopia, quimioterapia e radioterapia; podendo ocorrer, de forma habitual, a associação de mais de um destes métodos na mesma terapia proposta. Nos estágios avançados o tratamento combinado entre cirurgia e quimioterapia e/ou radioterapia são os mais recomendados (BATISTA TP, et al., 2013)

Em grande parte das pesquisas analisadas, os estudos defendem o pensamento de que a cirurgia ainda é a melhor opção de tratamento do câncer gástrico (ALVES JR, et al., 2011; BARCHI LC, et al., 2016; TONETO MG e VIOLA L, 2018). O estudo feito por Barchi LC et al. (2016), teve como objetivo principal analisar as vantagens das cirurgias minimamente invasivas nos estágios de câncer gástrico precoce. $O$ autor constatou que houve como benefícios principais o curto prazo de internação hospitalar (20,1\%), menor lesão cutânea e evita a possibilidade de que novas cirurgias sejam feitas em decorrência da primeira (BARCHI LC, et al., 2016).

Um estudo realizado por Kim H, et al. (2014), apontou que as cirurgias complexas foram sendo substituídas por métodos menos invasivos, como a gastrectomia laparoscópica, que neste período ainda estava em fase experimental. Após alguns anos de estudo é possível verificar que estes tipos de cirurgias estão cada vez mais presentes nos tratamentos gástricos (JUCÁ PC, et al., 2012). No estudo realizado por Alves JR, et al. (2011), a cirurgia minimamente invasiva é retratada, mediante a videolaparoscopia, como um fator importante na redução de complicações pós-cirúrgicas e melhora na qualidade de vida dos pacientes que são submetidos a este procedimento. Os autores expõem os pontos positivos e negativos, identificam que a abordagem menos invasiva, além de melhorar a morbimortalidade, aceleram a recuperação pós-operatória.

Outros artigos também reafirmam estes benefícios da cirurgia minimamente invasiva através da laparoscopia, apresentando seus pontos positivos como o menor risco de infecções e perda sanguínea, menor trauma, menores incisões cirúrgicas, realimentação precoce, retorno às atividades diárias precocemente, dentre outras vantagens. Com isso, evitam-se complicações, principalmente no que se refere ao melhor prognóstico. Relatam ainda que a laparoscopia, apesar de ser um procedimento mais recente, contém várias vantagens sobre o procedimento original, a laparotomia (BARCHI LC, et al., 2016; BATISTA TP, et al., 2013 e TAKATSU Y, et al., 2015). Segundo Zhou H, et al. (2015), há outra técnica terapêutica rápida, segura, minimamente invasiva, que resulta em um menor tempo operacional e menor perda sanguínea, chamado método Liga Sure. 
De acordo Barchi LC, et al. (2016), o Brasil realizou a primeira gastrectomia laparoscópica em 1993. Após mais de duas décadas, apenas alguns centros de saúde realizam o procedimento. Muitos são os coeficientes que tornam pouco viável a realização deste avanço terapêutico no Brasil, dentre eles pode-se citar as taxas exorbitantes que o governo reivindica para que haja acesso aos materiais importados necessários, o ensino do método não é corretamente propagado e esta terapêutica tem um custo elevadíssimo para o sistema público de saúde (BARCHI LC, et al., 2016).

Estudos citam a importância de uma decisão multidisciplinar no que tange a melhor forma terapêutica. As pesquisas pontuam também um melhor prognóstico em relação à terapia combinada do que somente a cirurgia, quimioterapia ou radioterapia (TAKATSU Y, et al., 2015; TONETO MG e VIOLA L, 2018). Segundo Takatsu Y, et al. (2015), um dos fatores que mais dificultam um prognóstico favorável para o câncer gástrico é sua incidência em pacientes cada vez mais jovens, resultando um diagnóstico tardio, já que esta patologia é mais comum em pacientes entre 50 a 70 anos. Porém, em comparação com os pacientes idosos, pode ser considerado um prognóstico favorável devido à baixa incidência de comorbidades associadas e complicações pós-operatórias (SHELLEY M, 2016).

Devido à alta porcentagem da população idosa asiática, metade dos casos de câncer gástrico ocorrem nestes países, sendo um dos motivos que levou Sakurai K et al. (2015), a realizarem uma pesquisa na qual tem como foco o esclarecimento da mortalidade operatória nos idosos. Estes pesquisadores verificaram que as comorbidades associadas, como doenças coronarianas, arritmia, doença cerebrovascular, Diabetes Mellitus, hipertensão, doença pulmonar e hepática, são os principais fatores para um prognóstico não tão favorável, concluindo que a gastrectomia é a mais ideal para uma melhor sobrevida (OMS, 2018). O continente Asiático é o de maior incidência desta patologia, sendo assim, o foco de pesquisa almejando a cura e/ou melhor prognóstico do câncer gástrico se encontra lá (SAKURAI K, et al., 2015).

Andreollo NA, et al. (2019), afirma que para obter êxito no tratamento e evitar recidivas da doença, é necessário realizar terapia conjunta, que consiste na junção do ressecamento gástrico, linfadenectomia e exploração detalhada dos linfonodos ressecados. A radioterapia mostrou-se bastante eficaz para concretização do tratamento. Tratamentos não convencionais, como as práticas alternativas, complementares e integrais, desde que comprovadas cientificamente a sua eficácia, podem ser utilizadas no combate ao câncer gástrico. Essas terapias complementam e integram o tratamento convencional contra o câncer $\mathrm{e}$ trazem maior conforto físico, espiritual e mental para os pacientes (AURELIANO WA, 2013).

A utilização de medicinas alternativas e complementares é parte do escopo social de pacientes oncológicos. A utilização de tais práticas tem um sentido sociocultural muito importante na construção da identidade destes pacientes, ajudando-os a decidirem em relação aos tratamentos, propiciando uma mudança no estilo e na qualidade de vida e influenciando positivamente no curso da doença. Entre essas práticas alternativas e complementares, cita-se a homeopatia, a medicina ayurvédica, a medicina tradicional chinesa, a medicina de ervas, as terapias psíquicas, as terapias espirituais e os grupos de apoio (SPADACIO $\mathrm{C} \mathrm{e}$ BARROS NF, 2008).

Observa-se que os tratamentos atuais e as descobertas terapêuticas que vêm ocorrendo são fundamentais na detecção precoce do câncer gástrico, melhorando consequentemente o prognóstico. Uma vez detectado precocemente, o tratamento será mais eficaz, pois facilita a redução e retirada do tumor, o que aumenta as chances de cura.

\section{CONSIDERAÇÕES FINAIS}

Evidenciou-se que a neoplasia gástrica faz parte das cinco neoplasias que mais leva ao óbito no mundo, sendo o continente asiático com maior número de incidência. No Brasil, a região mais acometida por esta patologia é a Norte, seguida do Nordeste, sendo o estado da Bahia, a quarta mais frequente e na cidade de Salvador é o sexto tipo mais incidente. Compreende-se a necessidade de melhora no estadiamento do câncer gástrico, a fim de aperfeiçoar as respostas terapêuticas, contribuindo para o aumento do prognóstico da sobrevida destes indivíduos para além de 5 anos. Tais resultados refletem um avanço na assistência prestada pela equipe de saúde, na aderência do paciente às alternativas de tratamento, reduzindo o número de óbitos. 


\section{REFERÊNCIAS}

1. ALVES JR, et al. Parcial Video-Assistida para Câncer Gástrico Precoce. ABCD Arq Bras Cir Dig, 2011; 24(3): 235238.

2. ANDREOLLO NA, et al. Adjuvant chemoradiotherapy after subtotal or total gastrectomy or and D2 lymphadenectomy increases survival in advanced gastric cancer?. ABCD Arq Bras Cir Dig., 2019; 32(4): 1-6.

3. AURELIANO WA. Terapias espirituais e complementares no tratamento do câncer: a experiência de pacientes oncológicos em Florianópolis (SC). Cad. Saúde Colet., 2013; 21(1): 18-24.

4. BARCHI LC, et al. Cirurgia Minimamente Invasiva no Câncer Gástrico Tempo de Mudar o Paradigma. ABCD Arq Bras Cir Dig, 2016; 29(2): 117-120.

5. BATISTA TP, et al. Perioperative Chemotherapy in Locally Advanced Gastric Cancer. Arq Gastroenterol, 2013; 50(3): 236-242.

6. BAÚ FA, HUTH A. Fatores de risco que contribuem para o desenvolvimento do câncer gástrico e de esôfago. Revista Contexto \& Saúde, ljuí, 2011; 11(21): 16-24.

7. CARVALHO JB, et al. Fatores de risco socioambientais e nutricionais envolvidos

8. na carcinogênese gástrica. Rev. para. Med, 2010; 25(2/3):1-9.

9. CHANG WK, et al. AssociationbetweenHelicobacterpyloriinfectionandtheriskofgastriccancer in the Korean population: prospective case-controlledstudy. Journal Of Gastroenterology, 2001; 36(12): 816-822.

10. COMISSÃO NACIONAL DE INCORPORAÇÃO DE TECNOLOGIAS NO SUS. Ministério da Saúde. DIretrizes Diagnósticas e Terapêuticas Adenocarcinoma de Estômago, 2017; 3 p.

11. DIACONU S, et al. Helicobacter Pylori Infection: old and new. Journal Of Medicine and Life, 2017; 10(2): $112-117$.

12. FERLAY J, et al. Cancer Incidence And Mortality Worldwide: sources, methods and major patterns in GLOBOCAN 2012. Int J Cancer, 2015; 136(5): 59-86.

13. FORD AC, et al. Helicobacter Pylori Eradication for the prevention of gastric neoplasia. Cochrane Database Syst Rev, 2015; 22(7): 1-45.

14. GONZÁLEZ CA, AGUDO A. Carcinogênese, prevenção e detecção precoce do câncer gástrico: onde estamos e para onde devemos ir. Int J Cancer, 2012; 130(4): 745-53.

15. INCA. 2018. In: O que é o Câncer?. 2018. Brasil.

16. JUCÁ PC, et al. Comparação da sobrevivência e dos fatores prognósticos em pacientes com adenocarcinoma gástrico T2 e T3. Rev Col Cir., 2012; 39(5): 377-384.

17. KIM H, et al. Long-Term Results of Laparoscopic Gastrectomy for Gastric Cancer: A Large-Scale Case-Control And Case-Matched Korean Multicenter Study. J Clin Oncol., 2014; 32(7): 627-633.

18. OMS. 2018. In: Folha Informativa - Câncer. Brasília.

19. RIBEIRO HF, et al. Cytogenetic characterization and evaluation of C-MYC gene amplification in PG100, a new Brazilian gastric cancer cell line. Braz. Journal Med Biol Res., 2010, 43(8): 717-721.

20. SAKURAI K, et al. The outcome of surgical treatment for elderly patients with gastric carcinoma. J Surg Oncol., 2015; 111(7): 848-854.

21. SANTOS MO. Estimativa 2018: Incidência de Câncer no Brasil. Revista Brasileira de Cancerologia, 2018; 64(1): 119120.

22. SHELLEY M. World Cancer Report 2014. Geneva, Switzerland: World Health Organization, International Agency for Research on Cancer, WHO Press, 2015. American Society for Nutrition, 2016; 7: 418-9.

23. SHEN L, et al. Management of gastric cancer in Asian: resource-stratified guidelines. The Lancet Oncology, 2013; 14(12): 535-547.

24. SONG H, et al. Molecular-targeted first-line therapy for advanced gastric cancer. Cochrane Database of Systematic Reviews, 2016; 7.

25. SPADACIO C, BARROS NF. Uso de medicinas alternativas e complementares por pacientes com câncer: revisão sistemática. Rev Saúde Pública, 2008; 42(1): 158-64.

26. STEWART BW, WILD CP. World Cancer Report: 2014. Ed. Lyon: IARC, 2014.

27. TAKATSU $Y$, et al. Clinicopathological features of gastric cancer in young patients. The International Gastric Cancer Association and The Japanese Gastric Cancer Association, 2015; 19: 472-478.

28. TONETO MG, VIOLA L. Estado Atual do Tratamento Multidisciplinar do Adenocarcinoma Gástrico. ABCD Arq Bras Cir Dig., 2018; 31(2): e 1373.

29. WANG Q, et al. Consumption of fruit, but not vegetables, may reduce risk of gastric cancer: results from a meta-analysis of cohort studies. Eur J Cancer, 2014; 50(8): 1498 - 1509.

30. WORLD HEALTH ORGANIZATION. 2018. In: International Agency for Research on Cancer. Cancer Today.

31. WORLD HEALTH ORGANIZATION. World Cancer Report 2014. International Agency for Research on Cancer, 2014; $632 \mathrm{p}$.

32. YAGHOOBI M, et al. Qual o risco quantitativo de câncer gástrico nos familiares de primeiro grau dos pacientes? Uma meta-análise. World J Gastroenterol., 2017; 23(13): 2435-42.32. YOON H, KIM N. Diagnóstico e manejo do grupo de alto risco para câncer gástrico. Fígado Intestino, 2015; 9(1): 5-17.

33. ZHOU H, et al. Short- and long-term outcomes of Ligasure versus conventional surgery for curative gastric cancer resection: a matched pair analysis. Gastric Cancer, 2015; 18: 843-849. 